\title{
Variable Red Giants in the LMC: Pulsating Stars and Binaries?
}

\author{
P. R. Wood \\ Research School of Astronomy and Astrophysics, Australian National University, \\ Private Bag, Weston Creek PO, Canberra ACT 2611, Australia \\ wood@mso.anu.edu.au \\ Received 1999 September 1, accepted 2000 January 7
}

\begin{abstract}
Infrared $J$ and $K$ photometry has been obtained of all red giants in the half square degree region of the LMC bar searched for variables using the MACHO database by Wood et al. (1999). The $K-\log P$ diagram shows the five distinct period-luminosity sequences which are also evident in the MACHO photometry. The $K-\log P$ plot allows identification of the Mira sequence from among the five observed sequences. As shown in Wood et al. (1999), comparison of observed periods, luminosities and period ratios with theoretical models identifies Miras unambiguously as radial fundamental mode pulsators, while semi-regular variables can be pulsating in the first, second or third overtone, or even the fundamental mode. All these variables lie on just three of the five distinct sequences, and they all appear to be on the AGB. The nature of the variability of stars on the other two sequences is currently unknown. Possibilities include contact and semi-detached binaries, rotating stars with giant star spots, episodic dust formation, rotationally stabilised non-radial $g^{-}$modes and strange pulsation modes caused by convection-pulsation interaction. These possibilities are discussed.
\end{abstract}

Keywords: binaries: close — Magellanic Clouds — stars: AGB and post-AGB stars: variables

\section{Introduction}

Red giant stars are probably the least understood of all variable stars. Although $o$ Ceti (Mira) was the first recorded pulsating star, and the Miras have by far the largest visual amplitudes of any class of pulsating star, even the mode of pulsation of these stars has remained a topic of controversy (e.g. Wood 1995; Bedding et al. 1998). All this has changed with the arrival of the MACHO survey of variable stars in the LMC: some problems have been solved, but unexpected new ones have turned up. In this paper, the results of the study of a complete sample of red giant stars in a $0.5 \times 0.5$ degree area of the LMC bar are discussed. A more detailed description of the MACHO data is given in Wood et al. (1999) and Alcock et al. (2000).

\section{Observed Period-Luminosity Sequences}

Infrared photometry in $J$ and $K$ was obtained on 7 December 1995 for all the giant stars in the half degree study area using the infrared camera CASPIR (McGregor et al. 1994) on the $2 \cdot 3 \mathrm{~m}$ telescope of the Australian National University. The $K$ magnitudes from these observations were combined with the periods of the variables obtained from $\mathrm{MACHO}$ data (Alcock et al. 2000) and plotted in the $(K, \log P)$

(C) Astronomical Society of Australia 2000 plane in Figure 1. Measurement errors in $K$ are estimated to be less than 0.03 magnitudes. Since the $K$ magnitudes are single-phase measurements, there will be a scatter introduced by variability of the stars. For the Mira variables, which are the largest amplitude long-period variables (LPVs), the total $K$ amplitude is $\sim 0.6$ mag. on average (Feast et al. 1982; Wood et al. 1983) so that the individual $K$ measurements for these stars might be expected to deviate from the mean by up to $0 \cdot 3$ magnitudes. For non-Mira LPVs, which have smaller amplitudes (see below), the deviations from the mean magnitude should be much less.

It has been known for many years that the Mira variables in the LMC lie on a single PL sequence in the $(K, \log P)$ plane (Feast et al. 1989; Hughes and Wood 1990). Furthermore, Wood and Sebo (1996) had found evidence for a second sequence at shorter periods [Bedding and Zijlstra (1998) have shown that the second sequence also appears to exist among the local semi-regular variables]. The Mira sequences of Feast et al. (1989) and Hughes and Wood (1990) are shown in Figure 1 and they clearly correspond with the sequence labelled $\mathrm{C}$. The largest amplitude variables also lie on sequence $\mathrm{C}$, providing more evidence that these stars are Mira variables. The second sequence of Wood and Sebo 


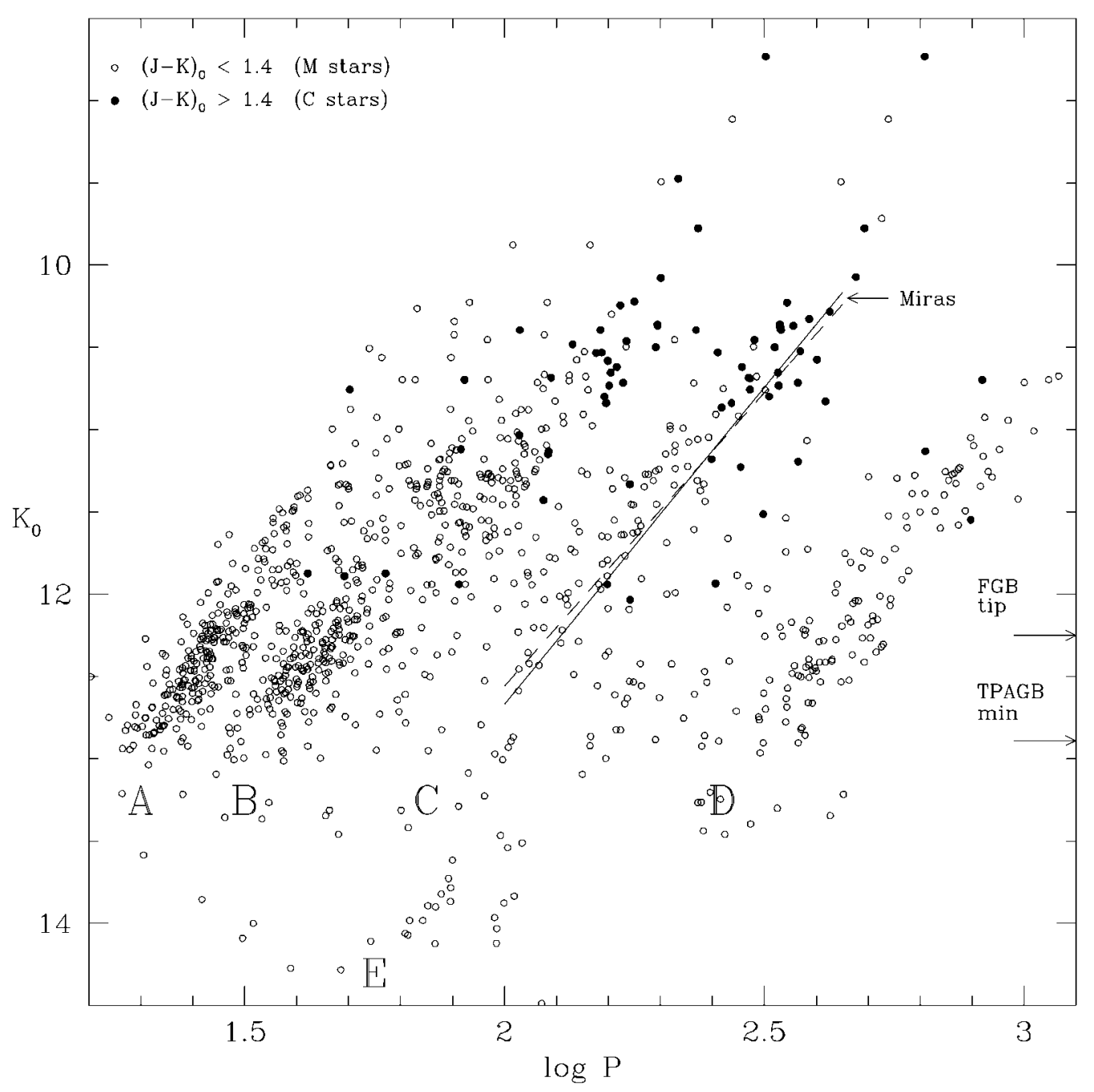

Figure 1-Variable LMC red giant stars plotted in the $(K, \log P)$ plane. Five sequences labelled A, B, C, D, E can be seen (see text). The positions of the tip of the first giant branch (FGB) and the minimum luminosity for thermally pulsing AGB (TPAGB) stars with mass $\sim 1 M_{\odot}$ are indicated by arrows. The solid and dashed lines are the $K-\log P$ relations from Hughes \& Wood (1990) and Feast et al. (1989) respectively. Solid circles correspond to stars with $J-K>1.4$ and they are assumed to be carbon stars. Other stars are assumed to be oxygen-rich M or K stars.

(1996) corresponds to the sequence labelled B in Figure 1. These stars are of smaller amplitude than many of those on sequence $\mathrm{C}$ and would be known as semi-regular (SR) variables in our Galaxy. Note, however, that many of the stars on sequence $\mathrm{C}$ are also of relatively small amplitude $(<1.5 \mathrm{mag}$. in $V)$ and would also be classified locally as SR variables. The stars of sequence A are of very low amplitude $(<0 \cdot 2$ mag. $)$ and would probably be classified locally as SARVs (small amplitude red variables): these stars were not previously known to fall on a well-defined PL sequence. In summary, the new MACHO data clearly highlight the previously-known PL sequences and show several additional sequences.

\section{The Pulsation Sequences}

Wood \& Sebo (1996) and Wood et al. (1999) have compared the observed PL relations with theoretical relations expected for radially pulsating asymptotic giant branch (AGB) stars. The comparisons show that sequence $\mathrm{C}$ (the Mira sequence) corresponds to pulsation in the fundamental mode while sequences $\mathrm{B}$ and $\mathrm{C}$ correspond to pulsation in the first, second and third overtones. A problem with these comparisons is that the effective temperatures (radii) of LPVs are poorly known so that it may be possible to fit a given pulsation mode to a different observed sequence by varying the assumed theoretical $T_{\text {eff }}$ (this will change the radius and hence the pulsation period). Wood et al. (1999) overcame this problem by using the fact that many of the LPVs in the MACHO sample are multimode pulsators so that period ratios in these stars could be plotted against the longer period (the so-called Petersen diagram) and then compared to theory. The only possible combination of pulsation mode and observed sequence which fitted both the PL laws and the Petersen diagram was the combination described above. It therefore 
seems that the identification of the fundamental pulsation mode as the mode of the Mira variables is now secure. This then raises the question of why the directly observed radii of Miras (e.g. Haniff et al. 1995; van Belle et al. 1996) are so large. The answer to this question is not currently known.

The most striking feature of Figure 1 is the group of four parallel period-luminosity (PL) sequences (A, B, C, D) brighter than $K \sim 13$ and the sequence $\mathrm{E}$ which consists of stars on the first giant branch (it also has an extension between sequences $\mathrm{C}$ and D-see Wood et al. 1999).

\section{Interpreting the Non-pulsation Sequences}

Adiabatic pulsation theory tells us that there is no mode of radial pulsation with a period longer that that of the fundamental mode. In Figure 1 it can be seen that the stars on sequence $\mathrm{D}$ have periods approximately 3-4 times longer than those of sequence C. Clearly, if it is accepted that sequence $\mathrm{C}$ corresponds to the fundamental radial pulsation mode, then the variability on sequence $\mathrm{D}$ cannot be attributed to radial pulsation.

Some examples of light curves of stars on sequence $\mathrm{D}$ are given in Wood et al. (1999). The oscillation period that fits sequence D is always a long secondary period (LSP), where the primary period usually falls on sequence B. The LSP amplitudes can be large, up to a factor of 2 in light output in the two MACHO bandpasses. The large amplitudes suggest that an explanation in terms of non-radial pulsation is unlikely ( $g$ modes could, in principle, have periods of the observed length). Similarly, although episodic dust formation of the type found by Winters et al. (1994) and Höfner et al. (1995) could cause the variability observed on sequence $\mathrm{D}$, most of the stars on this sequence are of relatively low luminosity and are not expected to have the high mass loss rates and dust-laden winds required for this process to occur. A third possible cause of the LSPs is rotation of red giants with a giant star spot. Once again, it seems difficult to image a spot sufficiently large to cause light variation by a factor of 2 , and it is not clear why the rotation period should be such as to cause the observed period-luminosity law.

At this stage, there are two seemingly plausible explanations for the LSPs on sequence D: binarity and strange pulsation modes. In the binary scenario, Wood et al. (1999) have shown that an orbiting companion at about two stellar radii from the red giant will have a period very similar to that observed in these stars. In order to produce the observed variability, the orbiting companion would need to carry along with it a large, loosely-bound, comet-like cloud which would partially obscure the red giant once per orbital period. The observational test of this scenario is to look for orbital radial velocity variations of the stars with LSPs. This is currently being done with $2 \mathrm{dF}$ on the AAT and it is estimated that companions with masses down to a few tenths of a solar mass should be detectable with these data. The results of this search for orbital velocity variations should be known by the end of this (second) millennium!

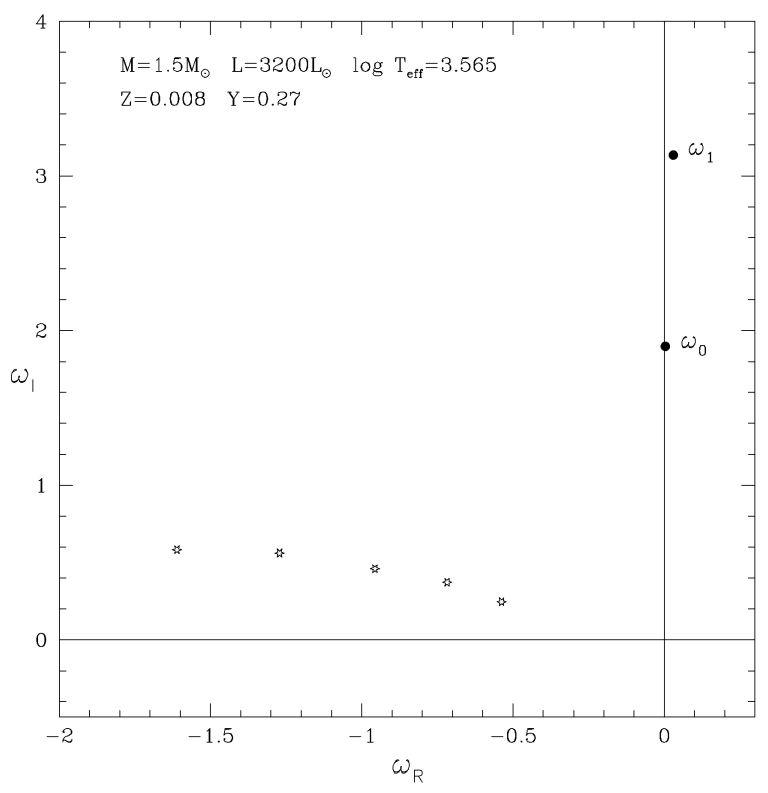

Figure 2-Eigenvalues in the complex $\omega$ plane for an AGB star with the parameters given on the figure. The linear, nonadiabatic, normal modes of pulsation for the fundamental $\left(\omega_{0}\right)$ and first overtone $\left(\omega_{1}\right)$ lie near the vertical axis. The eigenvalues of the first five COT modes are shown as starred symbols, starting near $\omega=(-0 \cdot 6,0 \cdot 3)$. All eigenvalues are normalised to the frequency $\left(G M / R^{3}\right)^{\frac{1}{2}}$.

The second plausible mechanism for the production of the LSPs is stellar oscillation caused by a newlydiscovered interaction of convection and pulsation. A study of the linear, non-adiabatic pulsation of red giants has shown the existence of a family of modes with just the periods observed on sequence D (Wood 1999). An investigation of the origin of these modes showed that they are a completely new type of oscillatory thermal mode caused by convection-pulsation interaction. The position of the eigenvalues $\omega$ of these convection-induced oscillatory thermal (COT) modes in the complex plane is shown in Figure 2, where a time dependence exp $\omega t$ has been assumed. Clearly, the frequencies of the COT modes (given by the imaginary part of $\omega$ ) are similar to those required to explain the LSPs. However, the extremely large damping of the modes (given by the real part of $\omega$ ) means that the COT modes found in these models will never be seen.

The treatment of convection-pulsation interaction used in the current models is very simplistic (it is based on mixing-length theory). It is not inconceivable that a proper treatment of convection-pulsation interaction could lead to unstable modes that could explain the LSPs. Why such modes should be seen 
in only $25 \%$ of AGB stars would then need to be answered. [Note: $25 \%$ is the percentage of LMC AGB variables that show LSPs; the LSPs also occur in local semi-regular variables (Houck 1963) but the percentage of local semi-regulars that show LSPs is not known because extended and high quality light curves are needed to find LSPs.]

The final sequence of stars in Figure 1 is sequence E. The stars on this sequence belong to the first giant branch (FGB) rather than the AGB. They can be picked out from a sample of LPVs by the regularity of their light curves, which are also of small amplitude (mostly less than a few tenths of a magnitude). Some examples are shown in Wood et al. (1999). A clue to the cause of the variability of these objects is provided by the fact that a few of them have light curves very similar to those of close eclipsing binaries or contact binaries. In addition, the periods are consistent with the orbital periods expected for contact binaries at the tip of the FGB. Once again, the $2 \mathrm{dF}$ observations of radial velocity variations should be able to confirm or refute this explanation for the origin of the variability of stars on sequence E.

\section{References}

Alcock, C., et al. 2000, in preparation

Bedding, T. R., \& Zijlstra, A. A. 1998, ApJL, 506, 47L
Bedding, T. R., Zijlstra, A. A., Jones, A., \& Foster, G. 1998, MNRAS, 301, 1073

Feast, M. W., Glass, I. S., Whitelock, P. A., \& Catchpole, R. M. 1989, MNRAS, 241, 375

Feast, M. W., Robertson, B. S. C., Catchpole, R. M., Lloyd Evans, T., Glass, I. S., \& Carter, B. S. 1982, MNRAS, 201, 439

Haniff, C. A., Scholz, M., \& Tuthill, P. G. 1995, MNRAS, 276,640

Höfner, S., Feuchtinger, M. U., \& Dorfi, E. A. 1995, A\&A, 297,815

Houck, N. 1963, AJ, 68, 253

Hughes, S. M. G., \& Wood, P. R. 1990, AJ, 99, 784

McGregor, P., Hart, J., Hoadley, D., \& Bloxham, G. 1994, in Infrared Astronomy with Arrays, ed. I. McLean (Dordrecht: Kluwer), p. 299

van Belle, G. T., Dyck, H. M., Benson, J. A., \& Lacasse, M. G. 1996, AJ, 112, 2147

Winters, J. M., Fleischer, A. J., Gauger, A., \& Sedlmayr, E. 1994, A\&A, 290, 623

Wood, P. R. 1995, in Astrophysical Applications of Stellar Pulsation, ed. R. S. Stobie \& P. A. Whitelock, ASP Conf. Ser. 83 (San Francisco: ASP), p. 127

Wood, P. R. 1999, in The Impact of Large-scale Surveys on Pulsating Star Research, IAU Colloquium 176, ed. L. Szabados \& D. Kurtz, ASP Conf. Series (San Francisco: ASP), in press

Wood, P. R., \& Sebo, K. M. 1996, MNRAS 282, 958

Wood, P. R., Bessell, M. S., \& Fox, M. W. 1983, ApJ, 272, 99

Wood, P. R., et al. 1999, in Asymptototic Giant Branch Stars, IAU Symposium 191, ed. T. Le Bertre et al. (San Francisco: ASP), p. 151 